\title{
Potential antitumor mechanisms of phenothiazine drugs
}

\author{
QI Lu ${ }^{1} \&$ DING YanQing ${ }^{1,2 *}$ \\ ${ }^{1}$ Department of Pathology, School of Basic Medical Sciences, Southern Medical University, Guangzhou 510515, China; \\ ${ }^{2}$ Department of Pathology, Nanfang Hospital, Southern Medical University, Guangzhou 510515, China
}

Received April 22, 2013; accepted September 10, 2013; published online October 14, 2013

\begin{abstract}
In this study, three kinds of phenothiazine drugs were analyzed to explore their potential antitumor mechanisms. First, target proteins that could interact with chlorpromazine, fluphenazine and trifluoperazine were predicted. Then, the target proteins of the three drugs were intersected. Cell signaling pathway enrichment and related disease enrichment were conducted for the intersected proteins to extract the enrichment categories associated with tumors. By regulation network analysis of the protein interactions, the mechanisms of action of these target proteins in tumor tissue were clarified, thus confirming the potential antitumor mechanisms of the phenothiazine drugs. The final results of cell signaling pathway enrichment and related disease enrichment showed that the categories with the highest score were all found in tumors. Target proteins belonging to the tumor category included signaling pathway members such as Wnt, MAPK and retinoic acid receptor. Moreover, another target protein, MAPK8, could indirectly act on target proteins CDK2, IGF1R, GSK3B, RARA, FGFR2 and MAPK10, thereby affecting tumor cell division and proliferation. Therefore, phenothiazine drugs may have potential antitumor effects, and tumorassociated target proteins play important roles in the process of cell signaling transduction cascades.
\end{abstract}

phenothiazines, antitumor, bioinformatics, target proteins

Citation: Qi L, Ding Y Q. Potential antitumor mechanisms of phenothiazine drugs. Sci China Life Sci, 2013, 56: 1020-1027, doi: 10.1007/s11427-013-4561-6

There are many types of antitumor chemotherapy drugs. According to the traditional classification system, they can be divided into alkylating agents, antimetabolites, antitumor antibiotics, plant-based, hormones and miscellaneous drugs. In turn, according to the effects of drugs on cell proliferation kinetics, they can be divided into cell cycle-specific drugs and cell cycle non-specific drugs. However, chemotherapy drugs have more side effects, such as nausea, vomiting, hair loss and pain, so many cancer patients undergo great suffering during this type of treatment. Phenothiazine drugs are relatively stable and widely used anti-psychotic drugs that mainly act on central dopamine receptors and have sedative, antiemetic, anti-psychotic and body temperature-lowering effects. Their representative drug is chlorpromazine. Studies have found that chlorpromazine can

*Corresponding author (email: dyq@ fimmu.com) enhance the cytotoxic effects of tamoxifen on breast cancer [1]. Phenothiazine drugs can also enhance the sensitivity of chemotherapy drugs such as cisplatin [2], and reverse resistance of tumor cells to chemotherapeutic drugs [3]. These results are indicative of the characteristics of their adjuvant chemotherapy. Other reports show chlorpromazine can specifically inhibit mitotic kinesin KSP/Eg5 to cause mitotic arrest, further inhibiting tumor cell proliferation [4], and can selectively exert cytotoxic effects on lymphoblastoid tumor, neuroblastoma, non-small-cell lung cancer and breast cancer cells relative to normal cells [5]. In studies of phenothiazine drugs to treat leukemia and lymphoma cells, it has been reported that a commonly used clinical dose of chlorpromazine and trifluoperazine can promote apoptosis in leukemia and lymphoma without affecting normal cells [6]. These evidences indicate that this type of drug can inhibit tumor proliferation. Previous studies have also found that 
phenothiazine drugs can inhibit tumor proliferation [7], and the probability of patients with schizophrenia developing cancer is lower than that of normal people [7,8], showing the potential antitumor effects of these drugs. Since phenothiazine drugs have varying degrees of antiemetic, sedative and analgesic effects, they may be able to reduce the side effects of chemotherapy, and they themselves also have a role in inhibiting tumor cell proliferation. These drugs can also reduce anxiety, insomnia and other symptoms related to the psychological state of cancer patients. Chlorpromazine was discovered in 1952, and has broad antitumor application prospects because of its common use and specific side effects. In this study, chlorpromazine and its derivatives, fluphenazine and trifluoperazine (Figure 1), were selected to predict drug target proteins. The tumor-associated target proteins that these drugs might act upon were further analyzed to clarify the possible antitumor mechanisms of these drugs.

\section{Materials and methods}

\subsection{Screening of antitumor drugs}

First, lung cancer gene expression profiling data (GSE10072 and GSE31210) and breast cancer gene expression profiling data (GSE29431 and GSE14548) were downloaded from the GEO Public Expression Profiling Database in National Center for Biotechnology Information (NCBI) [9]. Lung cancer is closely correlated with smoking, so lung tissue data associated with smoking were selected from lung cancer expression profile data. GSE10072 expression profile data included 34 normal lung tissues and 42 lung cancer tissues of patients who smoked. GSE31210 expression profile data included 12 normal lung tissues and 111 lung cancer tissues of patients who smoked. For the breast cancer expression profiles, GSE29431 data contained 12 normal breast tissues and 54 breast cancer tissues, and GSE14548 data contained 28 normal breast tissues and 38 breast cancer tissues. In the same expression profile, differ- entially expressed genes of normal tissues and tumor tissues were screened using Bonferroni correction [10]; results with $P$-values of $<0.05$ were selected. After screening, additional genes were screened from lung cancer tissues, so the conditions were further limited: changes in normal and tumor gene expression values were increased by two-fold. The expression profiles of both lung cancer and breast cancer contained two groups of expression profiling data submitted from different laboratories, so screened differentially expressed genes were intersected. Ninety-seven intersected genes were upregulated and 234 intersected genes were downregulated in lung cancer tissue compared with normal lung tissue, while 37 intersected genes were upregulated and 53 intersected genes were downregulated in breast cancer tissues compared with normal breast tissues. Differentially expressed gene-related drug enrichment analysis was converted to be conducted on intersected genes screened as described above using an Affymetrix U133A [11] probe and connectivity map [12]. The connectivity map was used to record the changing expression pattern profiles of tumor cells treated with a large number of small-molecule drugs; this screening tool was capable of matching expression profiles and chemotherapy drugs. Using this tool, tumor-associated small-molecule drugs could be screened by changes in tumor tissue expression profiles. According to these results, enriched fraction-negative drugs could reverse the expression direction of those expression profiles inputted. Inputted expression profile data included upregulated and downregulated differentially expressed genes, and the screened drugs could reverse this expression profiling mode, thus also potentially suppressing tumor occurrence and development. Therefore, drugs that enriched fractions were negative; $P$-values less than 0.05 and the number of experiments of compound taking effects on cells was larger than 8 were selected in the analysis. Enrichment fraction was negative, indicating that the compounds screened out had negative correlation with input expression profile. $P<0.05$ and eight experiments of compounds on cells all

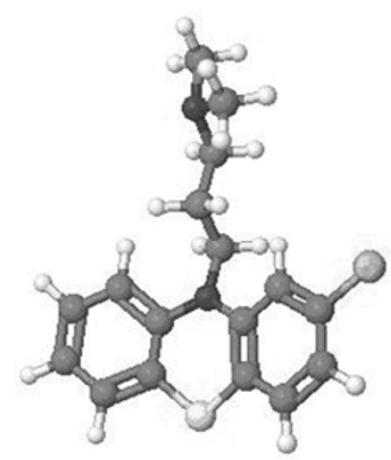

Chlorpromazine

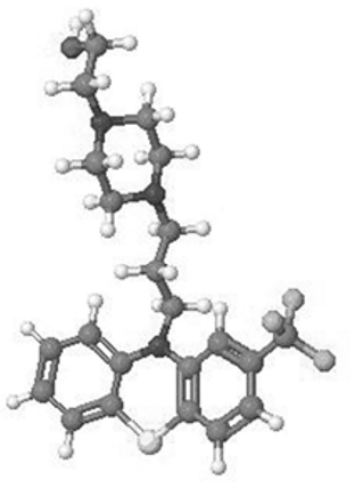

Fluphenazine

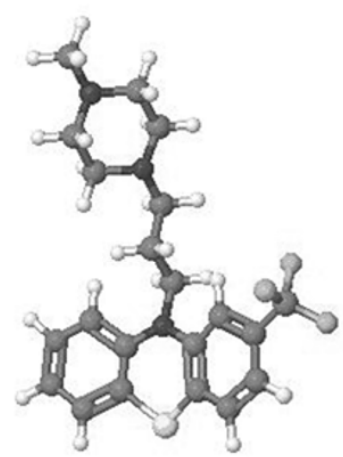

Trifluoperazine

Figure 1 Molecular structures of three kinds of phenothiazine drugs. The main structures of the three types of phenothiazine drugs are basically similar, while the side-chain radicals are different. Fluphenazine and trifluoperazine are all derivatives of chlorpromazine. 
suggested high validation. From the above analysis, we found that chlorpromazine, fluphenazine and trifluoperazine might have potential antitumor effects.

\subsection{Mechanism of action of phenothiazine drugs}

The DOCK6 database can simulate the docking of compounds and protein molecules to predict target proteins capable of interacting with compounds. Using DRAR-CPI (a server for predicting Drug Repositioning and Adverse Reaction via Chemical-Protein Interactome) [13] with DOCK6, target proteins capable of interacting with chlorpromazine, fluphenazine and trifluoperazine were predicted. The predicted target proteins of the three drugs were intersected to aggregate the target proteins that might be able to interact with all three drugs. Then, enrichment analysis was conducted on these target proteins through DAVID (Database for Annotation, Visualization and Integrated Discovery) [14] using the KEGG pathway database and gene-related disease classification database. Tumor-associated target proteins were taken from the enrichment result and protein interaction network analysis was conducted using the GeneMANIA [15] and Visant tools (Integrative Visual Analysis Tool for Biological Networks and Pathways) [16-18]. Cell signaling regulatory networks in which these target proteins were involved were analyzed, thus indicating the method by which the three drugs inhibit tumor cell proliferation. By matching the inputted genes in the database using DAVID, these genes were enriched in different categories according to various databases. GeneMANIA is a tool for rapid prediction of protein function, and can integrate multiple genomes and proteome data to infer proteins of unknown function. This tool can identify a plurality of protein functions by integrating information. Therefore, protein function-related modules in networks can be quickly found by using it to further tap the biological significance of the network. In contrast, the Visant tool mainly analyzes protein interactions to build networks using published protein interaction data.

\section{Results}

\subsection{Screening results of antitumor drugs}

Screening revealed that of the relevant drugs eventually selected against breast cancer (Table 1) and lung cancer (Table 2), chlorpromazine, fluphenazine and trifluoperazine belonged to the phenothiazine group of drugs, and fluphenazine and trifluoperazine were derivatives of chlorpromazine; the number of compounds having an effect on cells (n value) was high, indicating the results were relatively reliable. Subsequently, similar results were also obtained using the same method for expression profiles of colorectal cancer, indicating that these drugs probably had potential antitumor properties, consistent with previously reported findings. Thus we were confident that it provided a reliable basis for
Table 1 Ten drugs with potential antitumor effects in breast cancer based on expression profiling ${ }^{\text {a) }}$

\begin{tabular}{ccccc}
\hline Drug & Mean & $n$ & Enrichment & $P$ \\
\hline Prochlorperazine & -0.562 & 16 & -0.477 & 0.00076 \\
Chlorpromazine & -0.476 & 19 & -0.416 & 0.00168 \\
Trifluoperazine & -0.439 & 16 & -0.426 & 0.00383 \\
Resveratrol & -0.541 & 9 & -0.537 & 0.0058 \\
Deferoxamine & -0.458 & 8 & -0.541 & 0.01019 \\
0179445-0000 & -0.445 & 8 & -0.534 & 0.01161 \\
Alpha-estradiol & -0.458 & 16 & -0.376 & 0.01558 \\
Fluphenazine & -0.379 & 18 & -0.346 & 0.02103 \\
Methotrexate & -0.44 & 8 & -0.478 & 0.0338 \\
Thioridazine & -0.437 & 20 & -0.302 & 0.03949 \\
\hline
\end{tabular}

a) All $n$-values of the three phenothiazine drugs shown in Table 1 are relatively high, and all $P$-values are less than 0.05 , indicating they are likely to be associated with breast cancer.

Table 2 Ten drugs with potential antitumor effects in lung cancer screening based on expression profiling ${ }^{\text {a) }}$

\begin{tabular}{cccll}
\hline Drug & Mean & \multicolumn{1}{c}{$n$} & Enrichment & \multicolumn{1}{c}{$P$} \\
\hline Trichostatin A & -0.432 & 182 & -0.279 & 0 \\
Chlorpromazine & -0.449 & 19 & -0.46 & 0.00036 \\
LY-294002 & -0.394 & 61 & -0.257 & 0.0005 \\
Vorinostat & -0.552 & 12 & -0.515 & 0.00166 \\
Resveratrol & -0.554 & 9 & -0.554 & 0.00368 \\
Alpha-estradiol & -0.48 & 16 & -0.397 & 0.00874 \\
Tanespimycin & -0.327 & 62 & -0.201 & 0.01244 \\
Fluphenazine & -0.358 & 18 & -0.36 & 0.01443 \\
Thioridazine & -0.362 & 20 & -0.303 & 0.0384 \\
Trifluoperazine & -0.346 & 16 & -0.334 & 0.04225 \\
\hline
\end{tabular}

a) All $n$-values of the three phenothiazine drugs shown in Table 2 are relatively high, and all $P$-values are less than 0.05 , indicating they are likely to be associated with lung cancer.

the subsequent analysis.

\subsection{Predicting results of target proteins}

The target proteins of the three phenothiazine drugs were predicted using DRAR-CPI. These were calculated when the interaction strength of the drugs with target proteins reached a $Z$-score of less than -0.5 (combined with standardized score of strength). Ninety-eight target proteins that might be able to interact with chlorpromazine were predicted; 91 target proteins with fluphenazine and 117 target proteins with trifluoperazine. The three groups of target proteins described above were intersected, and a total of 39 target proteins that were likely to interact with all three types of phenothiazine drugs were obtained, thus improving the specificity of the interacting proteins.

\subsection{Enrichment of tumor-associated target proteins}

Enrichment analysis was conducted on the above-described 39 target proteins using the DAVID gene-related disease 
Table 3 Related disease enrichment of common target proteins of the three drugs ${ }^{\text {a) }}$

\begin{tabular}{|c|c|c|c|}
\hline Category & Term & Count & $P$-value \\
\hline GENETIC_ASSOCIATION_DB_DISEASE_CLASS & CANCER & 13 & 0.001877 \\
\hline GENETIC_ASSOCIATION_DB_DISEASE_CLASS & AGING & 5 & 0.00578 \\
\hline GENETIC_ASSOCIATION_DB_DISEASE_CLASS & REPRODUCTION & 6 & 0.023581 \\
\hline GENETIC_ASSOCIATION_DB_DISEASE_CLASS & DEVELOPMENTAL & 6 & 0.026687 \\
\hline GENETIC_ASSOCIATION_DB_DISEASE_CLASS & METABOLIC & 11 & 0.038986 \\
\hline GENETIC_ASSOCIATION_DB_DISEASE_CLASS & OTHER & 10 & 0.054997 \\
\hline GENETIC_ASSOCIATION_DB_DISEASE_CLASS & PHARMACOGENOMIC & 6 & 0.068428 \\
\hline GENETIC_ASSOCIATION_DB_DISEASE_CLASS & PSYCH & 8 & 0.072443 \\
\hline GENETIC_ASSOCIATION_DB_DISEASE_CLASS & HEMATOLOGICAL & 4 & 0.073614 \\
\hline GENETIC_ASSOCIATION_DB_DISEASE_CLASS & NEUROLOGICAL & 8 & 0.086108 \\
\hline
\end{tabular}

a) It can be seen from the enrichment results that tumor type shows the highest degree of significant association.

classification database. The results showed that the most relevant class was tumors, indicating that these target proteins were involved in tumor-associated biological processes (Table 3). Moreover, these phenothiazine drugs might be able to directly act on these target proteins, indicating that they could influence the occurrence and development of tumors. Pathway enrichment analysis was conducted on the target genes referred to above using the KEGG pathway database; tumor-related signaling pathways also showed the highest degree of association. Nine tumor-associated target proteins constituted this signaling pathway, namely CDK2, FGFR2, GSK3B, IGF1R, MAPK10, MAPK8, PPARG, RARA and RXRA (Table 4). CDK2 is a cell cycledependent protein kinase that participates in the regulation of the cell cycle, cell mitosis and phosphorylation of other tumor-associated proteins, and is thus closely associated with tumor growth. FGFR2 is an evolutionarily conserved fibroblast growth factor receptor involved in the regulation of cell proliferation, differentiation, migration and apoptosis, and embryonic development, and can mediate and activate the MAPK signaling pathway. FGFR2 overexpression can promote cell transformation and neoplasia. GSK3B, a glycogen synthase kinase with serine/threonine protein kinase activities, is an important molecule in the Wnt signaling pathway, which plays an important role in tumor development. IGF1R is an insulin-like growth factor receptor with tyrosine kinase activity that is overexpressed in most malignant tumors. It has anti-apoptotic effects and enhances cell survival. Both MAPK10 and MAPK8 belong to the mitogen-activated protein kinase family and are involved in a variety of cellular and molecular processes, including proliferation, differentiation, transcription regulation and development. They are also very closely related to tumors. PPARG is a member of the peroxisome proliferatoractivated receptor family and is involved in the regulation of adipocyte differentiation and glucose homeostasis. It is associated with various diseases, including obesity, diabetes, atherosclerosis and cancer. Studies have shown that PPARG is an independent marker for a good prognosis of colorectal cancer [19], and many studies have shown it has antitumor properties [20]. Both RARA and RXRA are important members of the retinoic acid signaling pathway and belong to the RARs and RXRs nuclear receptor families, respectively. RARA and RXRA play important roles in tumor genesis and development, which provide target point for chemotherapy drugs such as retinoic acid and are closely related to the efficiency of chemotherapy [21]. Therefore, they have been shown to be correlated with tumor chemotherapy. RARA can combine with RXRA to form a heterodimer involved in gene transcription and expression, while PPARG can also form a heterodimer with RXRA with similar functions [22]. Thus, PPARG, RARA and RXRA are closely linked in function.

\subsection{Analysis of network regulation of tumor-associated target protein interactions}

Network regulation analyses of protein interactions were conducted on the nine target proteins associated with tumor signaling pathways using GeneMANIA, as described above. It was found that $38.25 \%$ of target proteins and their interacting proteins have the same protein domain, indicating that these target proteins might have some similar functions in the tumor-associated signaling pathways, or are functionally interrelated. CDK2 and RARA proteins could colocalize, and MAPK8, MAPK10, RARA, RXRA, PPARG, FGFR2 and their interacting proteins had co-expression

Table 4 Target proteins associated with tumor-associated signaling pathways

\begin{tabular}{cc}
\hline Gene symbol & Gene name \\
\hline$C D K 2$ & Cyclin-dependent kinase 2 \\
$F G F R 2$ & Fibroblast growth factor receptor 2 \\
$G S K 3 B$ & Glycogen synthase kinase 3 beta \\
$I G F 1 R$ & Insulin-like growth factor 1 receptor \\
$M A P K 10$ & Mitogen-activated protein kinase 10 \\
$M A P K 8$ & Mitogen-activated protein kinase 8 \\
$P P A R G$ & Peroxisome proliferator-activated receptor gamma \\
$R A R A$ & Retinoic acid receptor, alpha \\
$R X R A$ & Retinoid X receptor, alpha \\
\hline
\end{tabular}




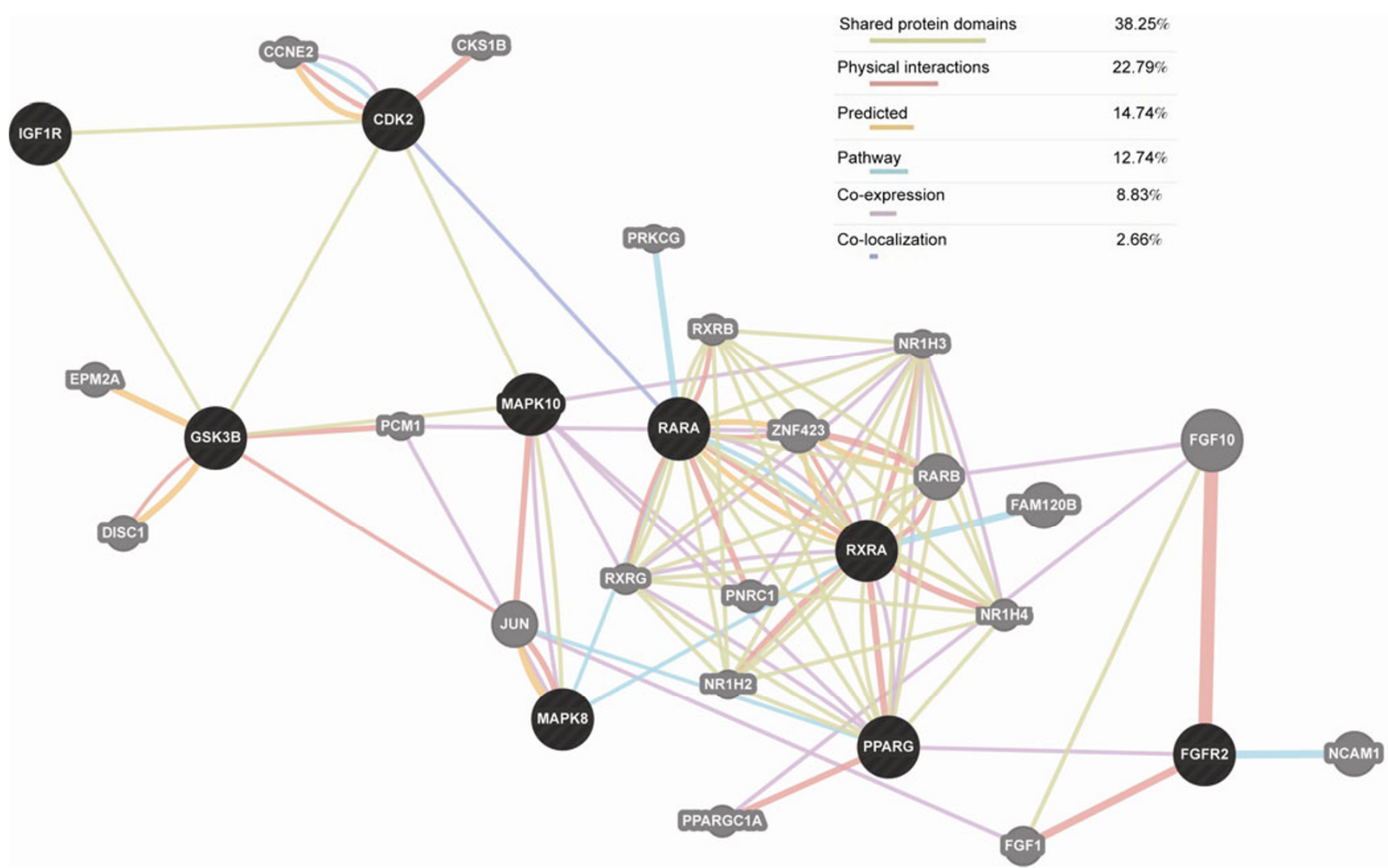

Figure 2 Tumor-associated target protein interactions. Black protein nodes indicate tumor-associated target proteins, while the gray nodes indicate target protein-related proteins; different connecting colors represent different correlations.

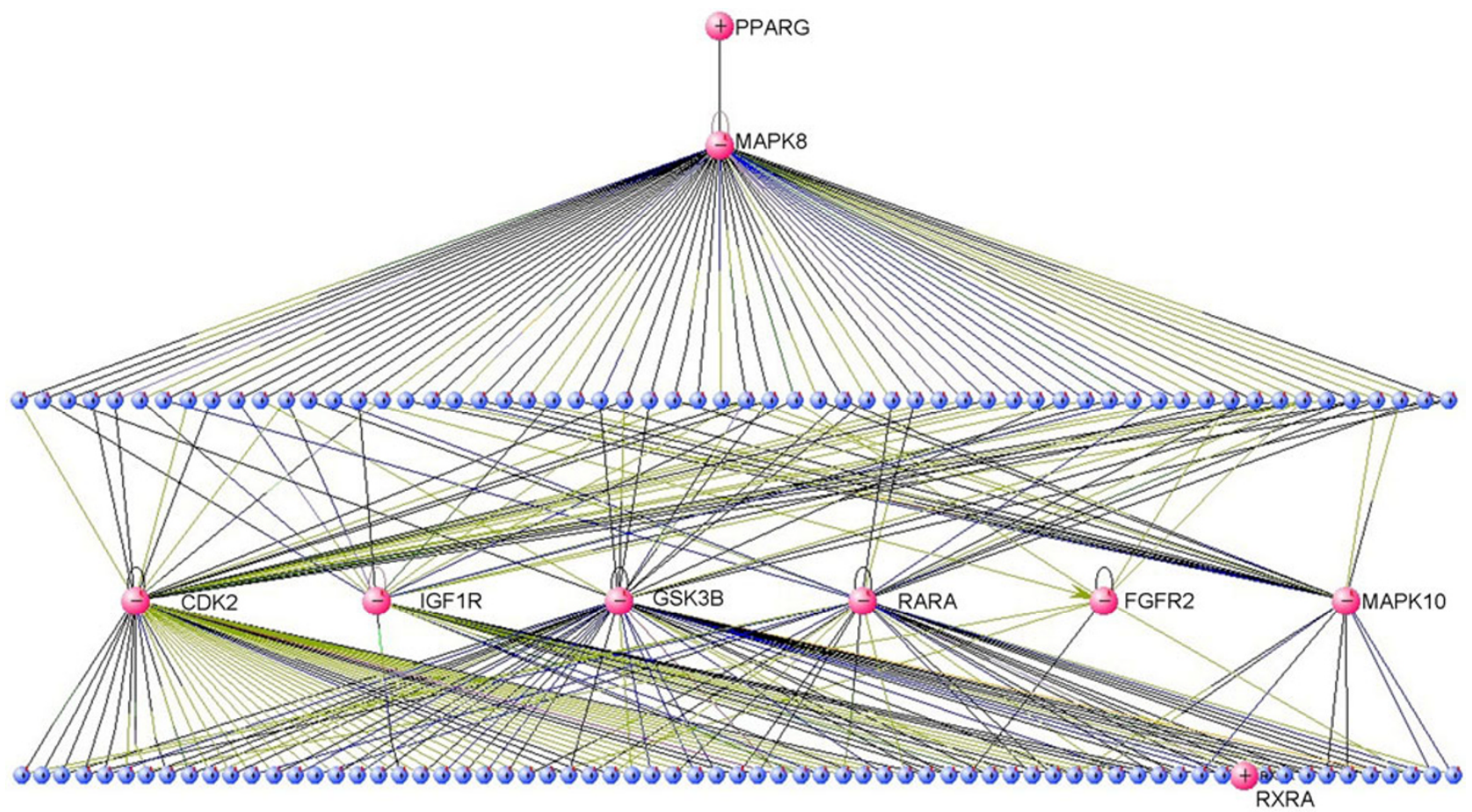

Figure 3 Network regulation of tumor-associated target protein interactions. Red nodes represent tumor-associated target proteins that could be affected by all three drugs, and blue nodes indicate the proteins that can interact with target proteins. Connecting lines between protein nodes represent the interactions among proteins. A connecting line with two or more colors illustrates the connection among proteins as supported by multiple experimental evidence. 
characteristics, further suggesting that these proteins have a common function. From the results of the network regulation of protein interactions, network modules composed of RARA, RXRA, PPARG and their interacting proteins were functionally closely linked, being possible key target proteins of phenothiazine drugs in the tumor cells. Furthermore, functional classes composed of this network module participate in retinoic acid signal transduction and may have certain correlations with cancer chemotherapy, further indicating that phenothiazines could enhance the antitumor property of chemotherapy drugs with auxiliary antitumor effects.

To further understand the effects of the nine identified target proteins associated with tumor signaling pathways, further analysis of interaction network regulation was conducted using the Visant tool. First, the target proteins were imported into Visant and the interacting proteins of each target protein were retrieved to identify any connections. Then, all network circuits among these nine target proteins were calculated, protein nodes that were not in circuits were removed, and signaling regulation networks dominated by these nine target proteins were obtained; by calculation, there were 128 protein nodes in the circuits. Enrichment analysis of the biological processes and molecular functions was conducted on the protein nodes in these circuits using WebGestalt. The results showed most of these 128 proteins have regulatory functions and are involved in metabolic processes and biological processes such as response to external stimuli, confirming these proteins are primarily involved in cell signal transduction. Therefore, the molecular function enrichment revealed that most of the proteins have binding functions; several proteins have nucleic acid binding abilities and transcriptional regulatory activity, indicating they are transcription factors. To specify how these proteins conduct cell signal transduction, re-combination of signal cascade regulation was conducted on these proteins to produce a network diagram of possible signal transduction regulation (Figure 3). From this, it appears that PPARG protein regulates MAPK8 protein. MAPK8 can directly affect 60 protein nodes in the upper layer, while these 60 protein nodes can affect six tumor-associated target proteins, which in turn can act upon 69 protein nodes in the lower layer, respectively. Of the 69 protein nodes, RXRA can be directly affected by RARA and GSK3B. This is a possible signal transduction cascade model that can specify how target proteins affect the signal transduction process; hence the molecular events in which the 60 blue protein nodes on the upper layer and the 69 blue protein nodes on the lower layer participated were analyzed further using GATHER (Gene Annotation Tool to Help Explain Relationships) [23]. The upper 60 protein nodes were mainly involved in the MAPK signaling pathway, and some protein nodes were also involved in apoptosis and cell cycle-related molecular events (Table 5), while the lower 69 protein nodes were mainly involved in cell metabolism, cell proliferation, gene transcription, mitosis and other cell proliferation-related molecular events (Table 6). From signal transmission to the final regulation of cell proliferation, the acting sites of three phenothiazine drugs were all important nodes in the process of cellular signal transduction, illustrating the critical role of the targets of these drugs in tumor development.

Table 5 Molecular events of the upper 60 blue protein nodes

\begin{tabular}{cc}
\hline Enrichment of biological processes of proteins on the upper layer & Number of proteins \\
\hline GO:0000165: MAPKKK cascade & 7 \\
GO:0007254: JNK cascade & 5 \\
GO:0007243: protein kinase cascade & 9 \\
GO:0006915: apoptosis & 10 \\
GO:0050794: regulation of cellular process & 13 \\
GO:0008629: induction of apoptosis by intracellular signals & 3 \\
GO:0007049: cell cycle & 12 \\
GO:0000074: regulation of cell cycle & 9 \\
GO:0007242: intracellular signaling cascade & 14 \\
\hline
\end{tabular}

Table 6 Molecular events of the lower 60 blue protein nodes

\begin{tabular}{cc}
\hline Enrichment of biological processes of proteins on the lower layer & Number of proteins \\
\hline GO:0006366: transcription from Pol II promoter & 15 \\
GO:0044238: primary metabolism & 54 \\
GO:0007049: cell cycle & 17 \\
GO:0000074: regulation of cell cycle & 12 \\
GO:0008283: cell proliferation & 19 \\
GO:0044237: cellular metabolism & 53 \\
GO:0006350: transcription & 26 \\
GO:0000910: cytokinesis & 7 \\
GO:0006351: transcription, DNA-dependent & 25 \\
\hline
\end{tabular}




\section{Discussion}

In this study, chemotherapy drugs related to breast cancer and lung cancer were selected based on differentially expressed genes identified through gene expression profiling; from this, we distinguished three phenothiazine drugs that may be able to inhibit breast cancer and lung cancer. Phenothiazine drugs are proposed to have potential antitumor effects, and similar results have been shown in colorectal cancer. In this study, the possible antitumor mechanisms of three phenothiazine drugs, chlorpromazine, fluphenazine and trifluoperazine, were further explored using bioinformatics methods. The target proteins of these drugs were predicted and found to be closely related to tumorassociated cell signaling. These proteins were further studied to construct regulatory protein networks using two network-building tools. Results of the GeneMANIA analysis showed that phenothiazine drugs may be able to act on signaling pathway members such as Wnt, MAPK and retinoic acid in tumor cells. All these target proteins are closely associated with tumors. A network module composed of RARA and RXRA target proteins may be relatively important. Because these target proteins are involved in retinoic acid signal transduction, they may be significant in chemotherapy. Through the Visant tool, the network described above was further refined and a model of the signal transduction cascade was constructed. From this, it can be seen that the target protein MAPK8 can indirectly impact upon target proteins CDK2, IGF1R, GSK3B, RARA, FGFR2 and MAPK10 through its interacting proteins, and these target proteins can directly affect cell proliferation, division and growth (Figure 4); thus, during signal transduction, the effects on some important nodes can further amplify the signals to alter the biological behaviors of the cells. However, whether protein interactions are promoted or inhibited requires further study. Because the mechanistic effects of the drugs on cell signaling are more complex, screening and analysis were conducted using a variety of methods in this study to improve the reliability of our conclusions. First, during the screening of tumor-associated chemotherapy drugs, phenothiazine drugs were found to have a high correlation with lung cancer, breast cancer and colorectal cancer, and in the analysis of the target proteins, because the molecular structures of three drugs are similar, the intersections of target proteins of three drugs were selected to improve the accuracy of the target proteins identified. Then, in the selection of the target proteins, those with strong interactions were selected for the intersection analysis. In the network regulation analysis of the target proteins, two tools were selected. These methods can be used to obtain more accurate conclusions and get a better handle on the antitumor mechanisms of phenothiazine drugs. Few studies have been conducted on the antitumor effects of phenothiazine drugs; therefore, this article provides new

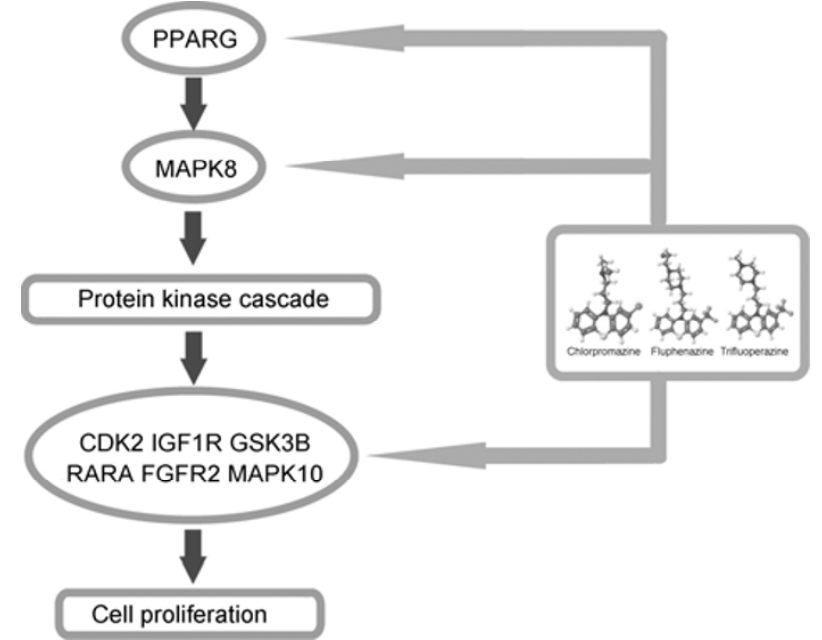

Figure 4 Possible antitumor mechanisms of three types of phenothiazine drugs. Tumor-associated signaling cascade as determined by bioinformatics analysis. Phenothiazine drugs might be able to interact with the identified protein nodes to inhibit the occurrence and development of tumors.

evidence for the antitumor effects of phenothiazine drugs. Of the phenothiazine drugs, chlorpromazine was discovered first. It can block dopamine receptors and has sedative, antiemetic and analgesic effects. As with other drugs, these kinds of drugs also have some adverse reactions such as dry mouth, fatigue, drowsiness, constipation and palpitations. A large quantity or long-term application of these drugs can cause extrapyramidal symptoms. However, because of the different functions of this drug, dosages are also inconsistent. Therefore, we should weigh up the advantages and disadvantages of their clinical use, and conduct close monitoring and dosage control to reduce the incidence of adverse reactions. The treatment of cancer is an important medical issue. Early tumor symptoms are often not obvious, causing many patients to present with distant organ metastasis upon diagnosis. Therefore, chemotherapy is an important means for treating tumors, particularly for patients with broad metastasis who are unsuitable candidates for surgery. However, the low specificity of chemotherapy drugs often results in unpleasant side effects. In this study, the potential antitumor mechanisms of three types of phenothiazine drugs were studied. This information may enable these drugs to be used rationally in tumor chemotherapy to reduce side effects and improve patient tolerance, thereby enhancing the inhibition efficiency of tumors and improving survival rates.

1 Yde C W, Clausen M P, Bennetzen M V, et al. The antipsychotic drug chlorpromazine enhances the cytotoxic effect of tamoxifen in tamoxifen-sensitive and tamoxifen-resistant human breast cancer cells. Anticancer Drugs, 2009, 20: 723-735

2 Eriksson A, Yachnin J, Lewensohn R, et al. DNA-dependent protein kinase is inhibited by trifluoperazine. Biochem Biophys Res Commun, 2001, 283: 726-731

3 Liang W, Yang C Z. Effects of phenothiazine derivatives on protein kinase C and multidrug resistance of tumor cells. Chin Sci Bull, 1998, 
43: 1179-1183

4 Lee M S, Johansen L, Zhang Y, et al. The novel combination of chlorpromazine and pentamidine exerts synergistic antiproliferative effects through dual mitotic action. Cancer Res, 2007, 67: 11359_ 11367

5 Wiklund E D, Catts V S, Catts S V, et al. Cytotoxic effects of antipsychotic drugs implicate cholesterol homeostasis as a novel chemotherapeutic target. Int J Cancer, 2010, 126: 28-40

6 Zhelev Z, Ohba H, Bakalova R, et al. Phenothiazines suppress proliferation and induce apoptosis in cultured leukemic cells without any influence on the viability of normal lymphocytes. Phenothiazines and leukemia. Cancer Chemother Pharmacol, 2004, 53: 267-275

7 Fond G, Macgregor A, Attal J, et al. Antipsychotic drugs: Pro-cancer or anti-cancer? A systematic review. Med Hypotheses, 2012, 79: $38-42$

8 Burgess D J. Anticancer drugs: Antipsychotic to anticancer agent? Nat Rev Drug Discov, 2012, 11: 516

9 Barrett T, Suzek T O, Troup D B, et al. NCBI GEO: Mining millions of expression profiles - database and tools. Nucleic Acids Res, 2005, 33: D562-D566

10 Shi Q, Pavey E S, Carter R E. Bonferroni-based correction factor for multiple, correlated endpoints. Pharm Stat, 2012, 11: 300-309

11 Orlov Y L, Zhou J, Lipovich L, et al. Quality assessment of the Affymetrix U133A\&B probesets by target sequence mapping and expression data analysis. In Silico Biol, 2007, 7: 241-260

12 Lamb J, Crawford E D, Peck D, et al. The Connectivity Map: Using gene-expression signatures to connect small molecules, genes, and disease. Science, 2006, 313: 1929-1935

13 Luo H, Chen J, Shi L, et al. DRAR-CPI: A server for identifying drug repositioning potential and adverse drug reactions via the chemical- protein interactome. Nucleic Acids Res, 2011, 39: W492-W498

14 Dennis G J, Sherman B T, Hosack D A, et al. DAVID: Database for annotation, visualization, and integrated discovery. Genome Biol, 2003, 4: P3

15 Warde-Farley D, Donaldson S L, Comes O, et al. The GeneMANIA prediction server: Biological network integration for gene prioritization and predicting gene function. Nucleic Acids Res, 2010, 38: W214-W220

$16 \mathrm{Hu}$ Z, Snitkin E S, Delisi C. VisANT: An integrative framework for networks in systems biology. Brief Bioinform, 2008, 9: 317-325

$17 \mathrm{Hu} \mathrm{Z}$, Mellor J, Wu J, et al. VisANT: Data-integrating visual framework for biological networks and modules. Nucleic Acids Res, 2005, 33: W352-W357

$18 \mathrm{Hu} \mathrm{Z}$, Mellor J, Wu J, et al. VisANT: An online visualization and analysis tool for biological interaction data. BMC Bioinformatics, 2004, 5: 17

19 Ogino S, Shima K, Baba Y, et al. Colorectal cancer expression of peroxisome proliferator-activated receptor gamma (PPARG, PPARgamma) is associated with good prognosis. Gastroenterology, 2009, 136: $1242-1250$

20 Girnun G. PPARG: A new independent marker for colorectal cancer survival. Gastroenterology, 2009, 136: 1157-1160

21 Clarke N, Germain P, Altucci L, et al. Retinoids: Potential in cancer prevention and therapy. Expert Rev Mol Med, 2004, 6: 1-23

22 Papi A, Rocchi P, Ferreri A M, et al. RXRgamma and PPARgamma ligands in combination to inhibit proliferation and invasiveness in colon cancer cells. Cancer Lett, 2010, 297: 65-74

23 Chang J T, Nevins J R. GATHER: A systems approach to interpreting genomic signatures. Bioinformatics, 2006, 22: 2926-2933

Open Access This article is distributed under the terms of the Creative Commons Attribution License which permits any use, distribution, and reproduction in any medium, provided the original author(s) and source are credited. 